\title{
The SMC super-shells as probes of the turbulent dynamics of the ISM
}

\author{
Itzhak Goldman ${ }^{1,2}$ \\ ${ }^{1}$ Department of Basic Sciences, Afeka Tel Aviv Academic College of Engineering, Tel Aviv, \\ Israel \\ ${ }^{2}$ Department of Astronomy and Astrophysics, School of Physics and Astronomy, Tel Aviv \\ University, Tel Aviv, Israel \\ email: goldman@wise.tau.ac.il
}

\begin{abstract}
The spatial power spectrum of the HI $21 \mathrm{~cm}$ intensity in the Small Magellanic Cloud (Stanimirovic et al. 1999) is a power law over scales as large as those of the SMC itself. It was interpreted as due to turbulence by Goldman (2000) and by Stanimirovic \& Lazarian (2001). The question is whether the power spectrum is indeed the result of a dynamical turbulence or is merely the result of a structured static density. In the turbulence interpretation of Goldman (2000) the turbulence was generated by the tidal effects of the last close passage of the LMC about 0.2 Gyr ago. The turbulence time-scale was estimated by Goldman to be 0.4 Gyr, so the turbulence has not decayed yet. Staveley-Smith et al. (1997) observed in the SMC about five hundreds of HI super shells. Their age is more than an order of magnitude smaller than the turbulence age. Therefore, if the turbulence explanation holds, their observed radial velocities should reflect the turbulence in the gas in which they formed. In the present work we analyze the observed radial velocities of the super shells. We find that the velocities indeed manifest the statistical spatial correlations expected from turbulence. The turbulence spectrum is consistent with that obtained by Goldman(2000).
\end{abstract}

Keywords. Turbulence, ISM, SMC, Super Shells

\section{Introduction}

The spatial power spectrum of the HI $21 \mathrm{~cm}$ intensity in the Small Magellanic Cloud was obtained by Stanimirovic et al. (1999). Interestingly, it is a power law over scales as large as that of the SMC itself. Similar power laws have been observed by Crovisier \& Dickey (1983) and by Green (1993) in the galaxy. The outstanding feature in the case of the SMC is the large scale of the observed correlations. The power laws signal underlying long range correlations in what looks like a field of random fluctuations of the intensity. For an optically thin medium along the line of sight, the $21 \mathrm{~cm}$ intensity is proportional to the column density. Therefore, the fluctuations in $21 \mathrm{~cm}$ intensity represent fluctuations in density.

A natural interpretation of the observed power spectra is that the underlying correlations in density fluctuations are due to a turbulence in which velocity fluctuations, that are coupled to density fluctuations, give rise to the observed power laws. The turbulence interpretation was suggested by Goldman (2000) and Stanimirovic \& Lazarian (2001).

Goldman (2000) suggested that this large scale turbulence was generated by instabilities in the bulk flows that resulted from the tidal interaction during the last close passage of the Large Magellanic Cloud (LMC) 2 Gyr ago (Gardiner \& Noguchi 1996). 
However, since the observations catch a snapshot of the intensity field and since the turbulence timescales are very long $(\sim 0.4 \mathrm{Gyr})$ one cannot rule out the possibility of a static correlated density field that reflects initial conditions.

In the present paper we propose a test to decide between these two alternatives.

\section{Analysis of the supershells radial velocity field}

Staveley-Smith et al. (1997) observed 501 HI super shells in the SMC. The proposed test relies on the fact that the timescale and age of the turbulence (if indeed there) are typically 1 to 2 orders of magnitude larger than the lifetimes of the super shells. Therefore, they have formed in the turbulent gas and their observed radial velocities should reflect the turbulent velocity field in the gas in which they were formed. We wish to look at them as markers registering the ambient gas velocity. If the radial velocity field exhibits spatial correlations consistent with those of the turbulence, assumed as responsible for the $21 \mathrm{~cm}$ intensity spectra, it will strengthen the case for dynamical turbulence as the source of the HI intensity power spectrum.

We use the data of the 501 super shells reported in Table 1 of Staveley-Smith et al. (1997). For each super shell, the residual radial velocity was found by subtracting from the observed velocity the large-scale best fit to a linear function of the coordinates,

$$
v_{i}=v_{o b s, i}-\left(c+s_{1} x_{i}+s_{2} y_{i}\right)
$$

with shell numbers $1 \leqslant i \leqslant 501$ where

$$
c=155.1 \mathrm{~km} \mathrm{~s}^{-1}, \quad s_{1}=12.34 \times 10^{-3} \mathrm{~km} \mathrm{~s}^{-1} \mathrm{pc}^{-1}, \quad s_{2}=4.46 \times 10^{-3} \mathrm{~km} \mathrm{~s}^{-1} \mathrm{pc}^{-1} .
$$

The coordinates of each shell $\left(x_{i}, y_{i}\right)$ are in units of pc and were obtained from the angular coordinates by adopting a distance of $60 \mathrm{kpc}$ to the SMC. The velocities are in units of $\mathrm{km} \mathrm{s}^{-1}$. The subtracted large scale velocity field is composed of a mean velocity and a term corresponding to a velocity gradient. The magnitude of the latter is consistent with values obtained by Gardiner \& Noguchi (1996).

We have computed the second order structure function and the autocorrelation for the residual velocity field along lines parallel to the coordinate axes. Interpolation was used to fit the discrete data along the lines to a continuous function. The different lines yielded similar results.

For simplicity, homogeneous and isotropic velocity field is assumed. In this case, the structure function and the autocorrelation depend only on the distance between the two points, $r=|\vec{r}|$. The structure function is

$$
S(r)=<\left(v\left(\overrightarrow{r^{\prime}}+\vec{r}\right)-v\left(\overrightarrow{r^{\prime}}\right)\right)^{2}>
$$

Similarly, the autocorrelation function is

$$
C(r)=<v\left(\overrightarrow{r^{\prime}}+\vec{r}\right) v\left(\overrightarrow{r^{\prime}}\right)>
$$

The angular brackets denote ensemble averaging. Assuming ergodicity, in addition to homogeneity and isotropy, ensemble averaging equals space averaging. As stated above, we use averages over lines so that

$$
S(l)=\frac{1}{L} \int_{0}^{L}(v(x+l)-v(x))^{2} d x
$$




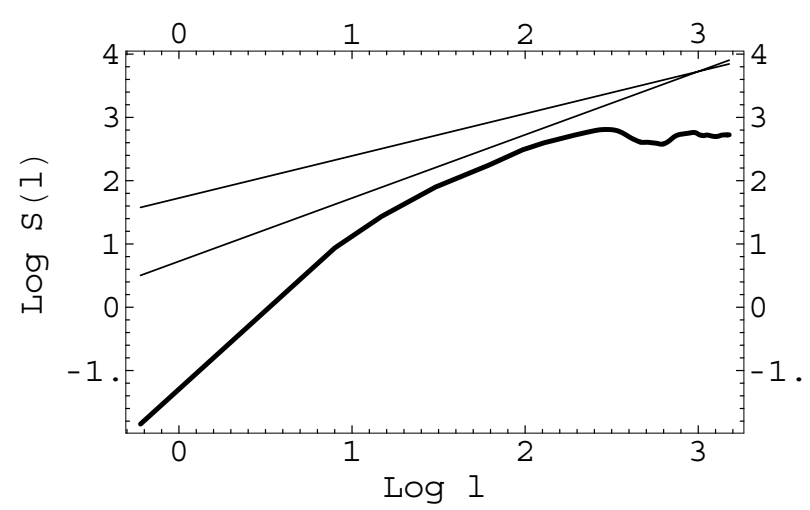

Figure 1. The structure function in units of $\left(\mathrm{km} \mathrm{s}^{-1}\right)^{2}$ as a function of scale in pc. The thin lines have slopes $2 / 3$ and 1 . The upper line has a slope of $2 / 3$.

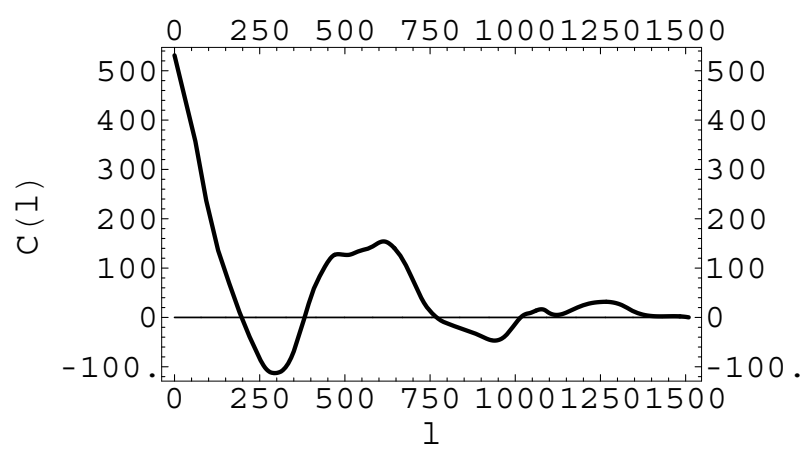

Figure 2. The autocorrelation function in units of $\left(\mathrm{km} \mathrm{s}^{-1}\right)^{2}$ as a function of scale in pc.

where $L$ is the length of the line. Similarly,

$$
C(l)=\frac{1}{L} \int_{0}^{L} v(x+l) v(x) d x
$$

The results of a typical computation are presented in figures 1-2. Figure 1 shows the structure function $S(l)$. For very small values of $l, S(l) \propto l^{2}$, for larger values of $l$ it varies as $S(l) \propto l^{m-1}$ and then it saturates.

The index $m$ characterizes the inertial range of the turbulent velocity spectral function: $F(k) \propto k^{-m}$. In Kolmogorov turbulence characterizing an incompressible fluid, $m=5 / 3$. In the case of turbulence in a compressible medium, $m=2$. This was also the value deduced by Goldman (2000) on the basis of the $21 \mathrm{~cm}$ intensity power spectrum. These two power laws are presented in figure 1. The precision of the data is not enough to decide between them, even though the $m=2$ line seems to follow better the slope of the computed structure function.

The autocorrelation function is shown in figure 2. It behaves as an autocorrelation function of a turbulent velocity rather than uncorrelated velocity fluctuations.

Figure 3 presents the turbulence spectral function $F(k)$ computed from the autocorrelation function. The curve is noisy but a power law range is clear. Also here the turbulence spectral functions with $m=5 / 3$ and $m=2$ are plotted. The two slopes are compatible with the computed spectral function, although $m=2$ seems preferable. 


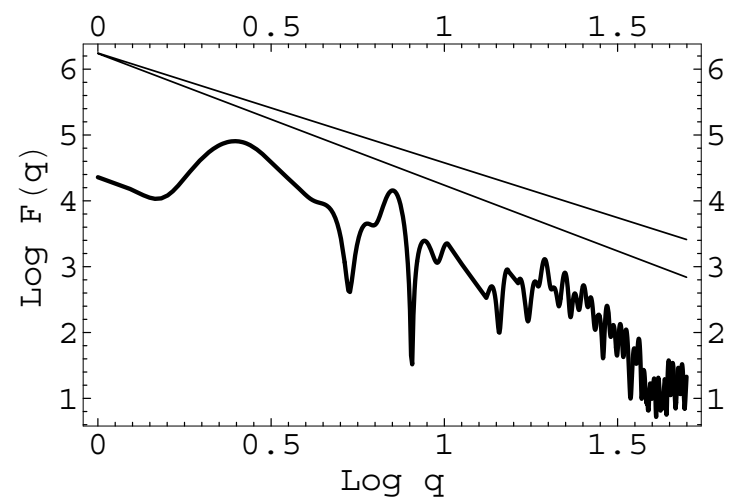

Figure 3. The turbulence spectral function in units of $\left(\mathrm{km} \mathrm{s}^{-1}\right)^{2} \mathrm{pc}$ as function of the normalized wavenumber $q=k L /(2 \pi)$. The upper thin line has a slope $-5 / 3$ and the lower -2 .

The wavenumber range shown corresponds to spatial scales between $1500 \mathrm{pc}$, which is in this case the length of the line $L$, and $50 \mathrm{pc}$. Higher wavenumbers correspond to spatial scales that are smaller than the average radius of the shells, and therefore the computed turbulence spectrum is not valid for these scales.

\section{Conclusions}

The results of the present work strengthen the case for the turbulence interpretation of the $21 \mathrm{~cm}$ power spectra of the SMC. The residual radial velocities of the super shells exhibit statistical spatial correlations expected from turbulence. The turbulence spectrum and structure function are consistent with a Kolmogorov spectrum, $m=5 / 3$, and with that of incompressible turbulence, $m=2$. The latter seems preferable. It equals the value deduced by Goldman (2000) from the HI intensity fluctuations.

\section{Acknowledgements}

Participation in IAU Symposium 237 was supported by Afeka Engineering College and by the Institute of Astronomy, Department of Astronomy and Astrophysics, Tel Aviv University.

\section{References}

Crovisier, J. \& Dickey, J. M. 1983, A\&A 122, 282

Gardiner, L. T. \& Noguchi, M. 1996, MNRAS 278,191

Goldman, I. 2000, ApJ 541, 701

Green, D. A. 1993, MNRAS 262, 327

Stanimirovic, S., Stavely-Smith, L., Dickey, J. M., Sault, R. J. \& Snowden, S. L. 1999, MNRAS 302,417

Staveley-Smith, L., Sault, R. J., Hatzidimitriou, D., Kesteven, M. J. \& McConnell, D. 1997, MNRAS 289, 225

Stanimirovic, S. \& Lazarian, A. 2001, ApJ 551, L53

\section{Discussion}

BLITz: How can you say that your mechanism is anything more than consistent with the observations, since your mechanism doesn't explain the turbulence in the outer regions of disks (beyond the stellar disks) or in spherical HI systems. 
Goldman: My talk referred to the particular case of the SMC. Particular in the sense that a tidal interaction with the LMC seems to be the plausible way to generate largescale shear flows who are unstable and generate turbulence. In any case, the main point was not identifying the energy source but showing that indeed the SMC is pervaded by large-scale velocity turbulence.

PADOAN: It is very difficult, from your velocity power spectrum plot, to evaluate if the power spectrum is $k^{-2}$ or $k^{-5 / 3}$. The quality of that HI velocity power spectrum is simply too poor to probe the turbulence.

Goldman: I agree. The index is $\sim(-5 / 3-2)$. But the important point is that radial velocities of the supershells exhibit a turbulence spectrum consistent with that deduced from the HI intensity power spectrum. 